\title{
Long non-coding RNA NEAT1 overexpression is associated with poor prognosis in cancer patients: a systematic review and meta-analysis
}

\author{
Chao Yang ${ }^{1}$, Zhuo Li ${ }^{1}$, Yajun Li ${ }^{2}$, Rui Xu ${ }^{3}$, Yongfeng Wang ${ }^{1}$, Yu Tian ${ }^{1}$, Wei Chen ${ }^{4}$ \\ ${ }^{1}$ Department of Clinical Laboratory, The First Affiliated Hospital of Xi'an Medical University, Xi'an City, Shaanxi Province, \\ China \\ ${ }^{2}$ Department of Neurology, The First Affiliated Hospital of Xi'an Medical University, Xi'an City, Shaanxi Province, China \\ ${ }^{3}$ Department of Clinical Research, The First Affiliated Hospital of Xi'an Medical University, Xi'an City, Shaanxi Province, China \\ ${ }^{4}$ Department of Clinical Laboratory, The First Affiliated Hospital of Xi'an Jiaotong University, Xi'an City, Shaanxi Province, \\ China
}

Correspondence to: Chao Yang, email: yangchaosup@126.com

Keywords: InCRNA, NEAT1, prognosis, cancer, meta-analysis

Received: October 09, $2016 \quad$ Accepted: November 24, $2016 \quad$ Published: December 01, 2016

\section{ABSTRACT}

Objectives: Long non-coding RNAs (IncRNAs) are playing important roles in cancer progression and metastasis. Recent studies have demonstrated that the IncRNA, nuclear paraspeckle assembly transcript 1 (NEAT1), was aberrantly up-regulated in various types of cancers and was reported to be associated with unfavorable prognosis in cancer patients. This study examined the relationship between NEAT1 and relevant clinical outcomes.

Results: A total of 1354 patients from 11 eligible studies were included in the meta-analysis. The results showed that high expression level of NEAT1 was significantly associated with shorter overall survival in cancer patients (hazard ratio $(\mathrm{HR})=1.53,95 \%$ confidence interval $(\mathrm{CI})=1.36-1.71)$; in the subgroup analysis, the positive association was also found in patients with hepato-gastroenterol cancers $(H R=1.79,95 \% C I=1.48-2.16)$, non-small cell lung cancer $(H R=1.35$, $95 \% \mathrm{CI}=1.04-1.76)$, ovarian cancer $(H R=1.41,95 \% \mathrm{CI}=1.11-1.79)$ and other types of cancers $(H R=1.42,95 \% \mathrm{CI}=1.11-1.81)$. The clinicopathological parameters analysis further showed that increased expression level of NEAT1 was positively correlated with larger tumor size (odds ratio $(O R)=1.74,95 \% \mathrm{CI}=1.26-2.41$ ), lymph node metastasis $(O R=2.29,95 \% \mathrm{CI}=1.71-3.06)$, advanced TNM stage $(\mathrm{OR}=3.60$, $95 \% \mathrm{CI}=2.27-5.72)$, poor tumor differentiation $(\mathrm{OR}=2.16,95 \% \mathrm{CI}=1.58-2.93)$, distant metastasis $(O R=3.51,95 \% \mathrm{CI}=1.75-7.01)$, and invasion depth $(O R=1.94$, 95\% CI = 1.36-2.75).

Materials and Methods: A comprehensive search was performed in Pubmed, Embase, Web of Science and CNKI databases, and eligible studies were included based on defined exclusion and inclusion criteria to perform meta-analysis.

Conclusions: The meta-analysis results from present study suggested that increased expression level of NEAT1 was associated with unfavorable prognosis and may serve as a predictive factor for clinicopathological features in various cancers.

\section{INTRODUCTION}

Cancer is becoming a major public health problem and is one of the main causes of morbidity and mortality worldwide [1]. The overall cancer-related death rates were still expected to rise in the future due to increased number of newly-diagnosed cases and insufficient understanding of the molecular mechanisms underlying cancer development [2]. In addition, the 5-year survival rate is still very low in many types of human cancers. 
Therefore, it is necessary for us to identify new potential biomarkers for early diagnosis and prognosis, and novel potential therapeutic target for the treatment of cancers.

Long non-coding RNAs (lncRNAs) are transcribed RNA molecules with more than 200 nucleotides and can not code proteins [3]. Many studies have demonstrated the diverse cellular functions of lncRNAs including cell proliferation, cell differentiation, cell apoptosis and carcinogenesis [4]. In the last decade, numerous studies have reported the dysregulation of lncRNAs in cancer, and the dysregulation of lncRNAs was found to contribute to cancer progression and metastasis. The lncRNAs such as H19 [5], Metastasis Associated Lung Adenocarcinoma Transcript 1 [6], HOX transcript antisense intergenic RNA [7], urothelial cancer associated 1 [8], antisense non-coding RNA in the INK4 locus [9] and PVT1 [10] were found to be novel promising biomarkers to predict a poor prognosis and lymph node metastasis in human cancers . Recently, the lncRNAs, nuclear paraspeckle assembly transcript 1 (NEAT1), was reported to have a role in cancer prognosis and chemo-/radio-sensitivity in a substantial number of studies. NEAT1 was found to be a diagnostic and prognostic biomarker in colorectal cancer [10], and $\mathrm{Li}$ et al., 2015 further showed that NEAT1 up-regulation is associated with tumor recurrence and unfavorable prognosis [11]. Study also showed that NEAT1 was identified as a critical modulator of prostate cancer by interacting with oestrogen receptor alpha [12]. In addition, NEAT1 enhances non-small cell lung cancer (NSCLC) via regulation of miR-377-3p-E2F3 pathway [13]. Fu et al., 2016 also found that NEAT1 was an unfavorable prognostic factor and promotes migration and invasion in gastric cancer [14].

Up to date, no meta-analysis has been performed to examine the relationship between NEAT1 and the relevant clinical outcomes. In the present study, relevant publications were collected to investigate whether the increased expression of NEAT1 could be served as a potential biomarker for prognosis in cancer patients.

\section{RESULTS}

\section{Study characteristics}

The detailed procedures of literature retrieval were shown in Figure 1. A total of 11 studies were finally identified. The total number of patients included in the present meta-analysis was 1354 , and the patient sample size ranges from 71 to 239 with a mean value of 96.0. Ten included studies were conducted in China, and one study was conducted in foreign countries. There are 8 type of cancers in the included studies, with two studies for ovarian cancer, two studies for colorectal cancer, two studies for NSCLC, one study for glioma, one study for nasopharyngeal carcinoma, one study for esophageal squamous cell carcinoma (ESCC), one study for hepatocellular carcinoma (HCC). All the clinical specimens were preserved before RNA extraction, and main information of the included studies were shown in Table 1.

\section{The association between NEAT1 expression levels and overall survival (OS)}

We first analyzed the association between NEAT1 expression levels and OS in the 11 included studies. The fixed-effects model was applied to estimate the pooled hazard ratios (HRs) and the respective 95\% confidence interval (CI). As show in Figure 2, there was no heterogeneity across these included studies $\left(\mathrm{P}_{\mathrm{h}}=0.73\right.$, $\left.\mathrm{I}^{2}=0 \%\right)$. The HR of the high NEAT1 expression level group versus the low NEAT1 expression level group was 1.53 (95\% CI $=1.36-1.71, P<0.001$, Figure 2 ). The results suggest that there was significant difference in the OS between high NEAT1 expression level group and low NEAT1 expression level group. A significantly shorter OS was shown in the patients with high NEAT1 expression level than that with low NEAT1 expression level. Thus, it is implied that the increased expression level of NEAT1 was associated with poor OS.

We further calculated the pooled HRs for OS based on different type of cancers. As shown in Figure 3, the effects of increased NEAT1 expression on OS was shown in patients with colorectal cancer $(\mathrm{HR}=1.91,95 \% \mathrm{CI}=1.41-2.58$, $P<0.001)$, NSCLC $(\mathrm{HR}=1.35,95 \% \mathrm{CI}=1.04-1.76$, $P=0.03)$, $\mathrm{HCC}(\mathrm{HR}=1.13,95 \% \mathrm{CI}=0.55-2.33, P=0.75)$, gastric cancer $(\mathrm{HR}=1.61,95 \% \mathrm{CI}=1.03-2.53, P=0.04)$, $\mathrm{ESCC}(\mathrm{HR}=1.92,95 \% \mathrm{CI}=1.40-2.63, P<0.001)$, glioma $(\mathrm{HR}=1.48,95 \% \mathrm{CI}=0.87-2.71, P=0.14)$, ovarian cancer $(\mathrm{HR}=1.41,95 \% \mathrm{CI}=1.11-1.79, P=0.004)$, and nasopharyngeal carcinoma $(\mathrm{HR}=1.39,95 \% \mathrm{CI}=1.06-1.83$, $P=0.02)$. In addition, we performed subgroup analysis based on cancer types, and similar results were obtained in hepatogastroenterol cancers $(\mathrm{HR}=1.79,95 \% \mathrm{CI}=1.48-2.16$, $P<0.001)$, NSCLC $((\mathrm{HR}=1.35,95 \% \mathrm{CI}=1.04-1.76$, $P<0.001)$, ovarian cancers $((\mathrm{HR}=1.41,95 \% \mathrm{CI}=1.11-1.79$, $P=0.004)$ and other types of grouped cancers ( $\mathrm{HR}=1.42$, $95 \% \mathrm{CI}=1.11-1.81, P=0.001$ ) (see Table 2 for details and Supplementary Figure S1 for Forest plot).

Further, we also performed subgroup meta-analysis stratified by analysis type, sample size and cut-off value, and similar results were found in regard the effects of increased NEAT1 expression level on OS (See Table 2 for details and see Supplementary Figures S2, S3 and S4 for the Forest plot).

\section{The association between NEAT1 expression level and disease-free survival (DFS) and progression- free survival (PFS) in cancer patients}

In the included study, there was only one study showed the association between NEAT1 expression level 
Table 1: Summary of all included eligible studies

\begin{tabular}{|c|c|c|c|c|c|c|c|c|c|c|}
\hline First Author & Year & Cancer type & $\begin{array}{c}\text { Total } \\
\text { number }\end{array}$ & Tumor stage & $\begin{array}{c}\text { Follow-up } \\
\text { (months) }\end{array}$ & $\begin{array}{c}\text { Adjuvant therapy } \\
\text { before surgery }\end{array}$ & $\begin{array}{c}\text { Criterion of high } \\
\text { expression }\end{array}$ & $\begin{array}{c}\text { Detection } \\
\text { method }\end{array}$ & $\begin{array}{l}\text { Outcome } \\
\text { measures }\end{array}$ & $\begin{array}{c}\text { Multivariate } \\
\text { analysis }\end{array}$ \\
\hline Chen ZJ [28] & 2016 & Ovarian cancer & 149 & 53/96(I-II/III-IV) & Over 60 & None & Median expression & qRT-PCR & OS & Yes \\
\hline Fu JW [14] & 2016 & Gastric cancer & 140 & 63/77(I-II/III-IV) & Over 60 & NR & Median expression & qRT-PCR & OS & Yes \\
\hline Sun C [13] & 2016 & NSCLC & 96 & 28/68 (I-II/III/IV) & Over 40 & None & NR & qRT-PCR & OS & No \\
\hline Adriaens C [19] & 2016 & Ovarian cancer & 58 & NR & Over 60 & Yes & NR & qRT-PCR & OS, PFS & No \\
\hline Guo S [29] & 2016 & $\mathrm{HCC}$ & 95 & 22/73(I-II/III-IV) & Over 60 & NR & Median expression & qRT-PCR & os & No \\
\hline $\operatorname{LiY}[11]$ & 2015 & Colorectal cancer & 239 & 92/147(I-II/III-IV) & Over 60 & None & Fold change & qRT-PCR & OS, DFS & Yes \\
\hline $\mathrm{He}$ C [30] & 2015 & Glioma & 94 & 23/71 (I-II/III-IV) & Over 50 & None & Median expression & qRT-PCR & OS & Yes \\
\hline Pan LJ [31] & 2015 & NSCLC & 125 & 54/71(I-II/III-IV) & Over 40 & NR & Mean expression & qRT-PCR & OS & No \\
\hline Chen X [32] & 2015 & ESCC & 96 & 35/61 (I-II/III-IV) & Over 60 & None & Youden index & qRT-PCR & os & Yes \\
\hline Wu Y [33] & 2015 & Colorectal cancer & 191 & 26/165 (I-II/III-IV) & Over 60 & None & Mean expression & qRT-PCR & OS & Yes \\
\hline Lu Y [34] & 2015 & $\begin{array}{l}\text { Nasopharyngeal } \\
\text { carcinoma }\end{array}$ & 71 & 36/35(I-II/III-IV) & Over 40 & NR & NR & qRT-PCR & OS & No \\
\hline
\end{tabular}

Abbreviations: DFS = disease-free survival; $\mathrm{ESCC}=$ esophageal squamous cell carcinoma; $\mathrm{HCC}=$ hepatocellular carcinoma; $\mathrm{NR}=$ not reported; NSCLC $=$ non-small cell lung cancer; OS = overall survival.

and DFS, and one study for association between NEAT1 and PFS. The results of HR showed as high NEAT1 expression versus low NEAT1 expression for DFS in patients with colorectal cancer was $1.80(95 \% \mathrm{CI}=1.27-2.55$, $P<0.001)$, and for PFS in patient with ovarian cancer was $2.99(95 \% \mathrm{CI}=1.21-4.36, P=0.011)$, which indicated a significantly positive association between high expression level of NEAT1 and poor DFS or poor PFS. However, as one study was performed, the meta-analysis was not performed.

\section{The association between NEAT1 expression levels and clinicopathological parameters}

In order to examine if NEAT1 expression had an association with clinicopathological parameters, we pooled the clinicopathological data for the meta-analysis. As shown in Table 3, the increased NEAT1 expression was significantly associated with larger tumor size (odds ratio $(\mathrm{OR})=1.74$, 95\% CI $=1.26-2.41, P<0.001$, Supplementary Figure S8), lymph node metastasis $(\mathrm{OR}=2.29,95 \% \mathrm{CI}=1.71-3.06$,

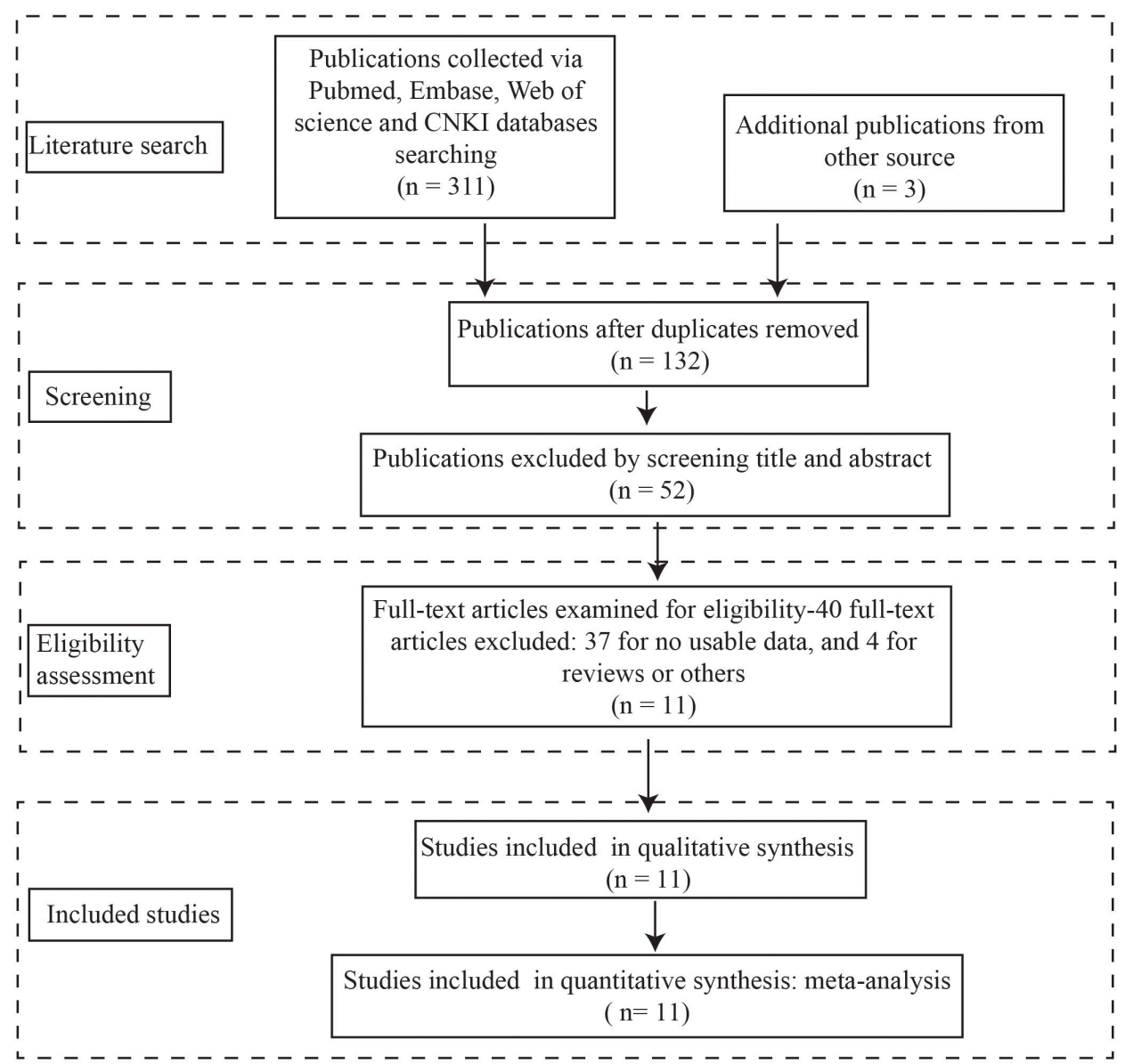

Figure 1: Stepwise procedures for searching databases and selecting eligible studies. 
Table 2: Subgroup meta-analysis of pooled HRs for OS

\begin{tabular}{|c|c|c|c|c|c|c|}
\hline \multirow{2}{*}{ Categories } & \multirow{2}{*}{ Studies (n) } & \multirow{2}{*}{$\begin{array}{c}\text { Number of } \\
\text { patients }\end{array}$} & \multicolumn{2}{|c|}{ Fixed-effects model } & \multicolumn{2}{|c|}{ Heterogeneity } \\
\hline & & & HR $(95 \% \mathrm{CI})$ for $\mathrm{OS}$ & $P$-value & $I^{2}(\%)$ & $P_{\mathrm{h}}$ \\
\hline [1] OS & 11 & 1354 & $1.53(1.36-1.71)$ & $<0.001$ & 0 & 0.73 \\
\hline \multicolumn{7}{|l|}{ [2] Cancer type } \\
\hline $\begin{array}{l}\text { 1) Hepato- } \\
\text { gastroenterol cancers }\end{array}$ & 5 & 761 & $1.79(1.48-2.16)$ & $<0.001$ & 0 & 0.65 \\
\hline 2) NSCLC & 2 & 221 & $1.35(1.04-1.76)$ & 0.03 & 0 & 0.75 \\
\hline 3) Ovarian cancer & 2 & 207 & $1.41(1.11-1.79)$ & 0.004 & 0 & 0.97 \\
\hline 4) others & 2 & 165 & $1.42(1.11-1.81)$ & 0.006 & 0 & 0.76 \\
\hline \multicolumn{7}{|l|}{ [3] Analysis type } \\
\hline Multivariate & 6 & 909 & $1.76(1.46-2.11)$ & $<0.001$ & 0 & 0.72 \\
\hline Survival curves & 5 & 445 & $1.39(1.20-1.61)$ & $<0.001$ & 0 & 0.99 \\
\hline \multicolumn{7}{|l|}{ [4] Sample sizes } \\
\hline$\geq 100$ & 5 & 844 & $1.68(1.35-2.09)$ & $<0.001$ & 0 & 0.69 \\
\hline$<100$ & 6 & 510 & $1.48(1.29-1.69)$ & $<0.001$ & 0 & 0.58 \\
\hline \multicolumn{7}{|l|}{ [5] Cut-off values } \\
\hline Mean & 2 & 316 & $1.67(1.10-2.55)$ & 0.02 & 46 & 0.17 \\
\hline Median & 4 & 478 & $1.47(1.11-1.96)$ & 0.008 & 0 & 0.87 \\
\hline Others & 5 & 560 & $1.53(1.34-1.74)$ & 0.001 & 5 & 0.38 \\
\hline
\end{tabular}

$P<0.001$, Supplementary Figure S9), advanced TNM stage $(\mathrm{OR}=3.60,95 \% \mathrm{CI}=2.27-5.72, P<0.001$, Supplementary Figure $\mathrm{S} 10)$, poor tumor differentiation $(\mathrm{OR}=2.16,95 \%$ $\mathrm{CI}=1.58-2.93, P<0.001$, Supplementary Figure S11), distant metastasis $(\mathrm{OR}=3.51,95 \% \mathrm{CI}=1.75-7.01$, $P<0.001$, Supplementary Figure S12), and higher invasion depth $(\mathrm{OR}=1.94,95 \% \mathrm{CI}=1.36-2.75, P<0.001$, Supplementary Figure S13). No significant correlation was observed between the increased NEAT1 expression with age, gender, and smoking status (see Table 3 for the details and see Supplementary Figures S5, S6 and S7 for the Forest plots). Because of the insufficient data for other clinicopathological parameters (such as recurrence, lymphatic invasion), the relationship between increased NEAT1 expression level and these clinicopathological parameters were not processed for the meta-analysis.

\section{Sensitivity analysis}

For the meta-analysis of the association between NEAT1 expression level and OS, the sensitivity analysis was performed by removing each study in turn from the

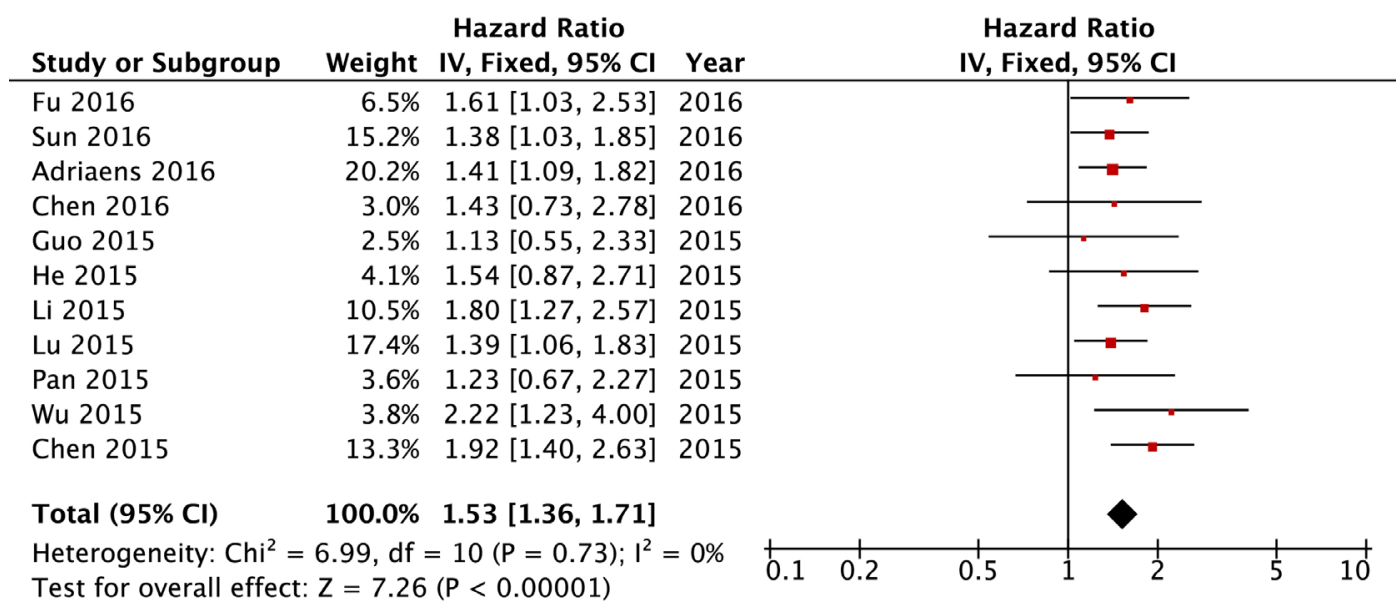

Figure 2: Forest plot of HRs for the association between high NEAT1 expression and OS in cancer patients. 
Table 3: Meta-analysis of association between increased NEAT1 expression and clinicopathological parameters

\begin{tabular}{|c|c|c|c|c|c|c|c|}
\hline \multirow{2}{*}{ Clinicopathological parameters } & \multirow{2}{*}{ Studies ( $n$ ) } & \multirow{2}{*}{ Patients $(n)$} & \multirow{2}{*}{ OR (95\% CI) } & \multirow{2}{*}{$P$-value } & \multicolumn{3}{|c|}{ Heterogeneity } \\
\hline & & & & & $I^{2}(\%)$ & $\mathbf{P}_{\mathrm{h}}$ & Model \\
\hline Age $(\geq 55$ vs. $<55$ years $)$ & 7 & 945 & $0.95(0.73-1.25)$ & 0.74 & 0 & 0.65 & Fixed \\
\hline Gender (Male vs. Female) & 6 & 796 & $0.96(0.72-1.28)$ & 0.77 & 40 & 0.14 & Fixed \\
\hline Smoking (Yes vs. No) & 2 & 227 & $0.96(0.54-1.71)$ & 0.9 & 0 & 0.61 & Fixed \\
\hline Tumor size $(\geq 5 \mathrm{~cm}$ vs. $<5 \mathrm{~cm})$ & 5 & 677 & $1.74(1.26-2.41)$ & $<0.001$ & 0 & 0.41 & Fixed \\
\hline Lymph node metastasis (Yes vs. No) & 6 & 806 & $2.29(1.71-3.06)$ & $<0.001$ & 18 & 0.3 & Fixed \\
\hline TNM stage (III-IV vs. I-II) & 7 & 945 & $3.60(2.27-5.72)$ & $<0.001$ & 58 & 0.03 & Random \\
\hline $\begin{array}{l}\text { Tumor differentiation } \\
\text { (Poor vs. Moderate/Well) }\end{array}$ & 5 & 334 & $2.16(1.58-2.93)$ & $<0.001$ & 0 & 0.77 & Fixed \\
\hline Distant metastasis (Yes vs. No) & 5 & 755 & $3.51(1.75-7.01)$ & $<0.001$ & 61 & 0.04 & Random \\
\hline Invasion depth (T3-T4 vs. T1-T2) & 3 & 276 & $1.94(1.36-2.75)$ & $<0.001$ & 0 & 0.49 & Fixed \\
\hline
\end{tabular}

pooled analysis. This analysis functions to examine the impact of the removed study on the overall HRs. In the present study, removing any of the included studies had no significant influence on the results, which suggests the robustness of the results.

\section{Analysis of publication bias}

In order to assess whether publication bias was existed in the included studies regarding the association between NEAT1 expression and OS, we performed the funnel plot analysis and applied the trim and filled methods (Figure 4), and our results demonstrated that on obvious publication bias was presented in the included studies for meta-analysis.

\section{DISCUSSION}

NEAT1 is a $\sim 3.2 \mathrm{~kb}$ novel nuclear long non-coding RNA, located in chromosome 11q13.1 [15]. Recent studies identified NEAT1 as a crucial architectural component of a paraspeckle structure, and NEAT1 has been shown to regulate numerous biological processes including cellular differentiation and stress response through paraspeckles pathway [15-17]. In the cancer studies, NEAT1 was found to be up-regulated in various types of cancer tissues and cancer cell lines, and NEAT1 was a key mediator in cancer progression by the regulation of cell apoptosis, cell proliferation as well as cell cycle [18]. In this regard, NEAT1 has been suggest as a potential diagnostic marker and may represent a novel target for the treatment of cancers. Apart from this, NEAT1 was also found to contribute to the chemo-/radio-resistance in ovarian cancer, lung cancer and nasopharyngeal carcinoma, which implicated that NEAT1 could be a potential biomarker for chemo-sensitivity.
A lot of efforts have been made to understand the functional role of NEAT1 in cancer progression, but the underlying molecular mechanisms of NEAT1 involved cancer progression are largely unknown. An important recent study demonstrated that NEAT1-containing paraspeckles could be induced by p53 which in turn modulates the replication stress and chemosensitivity in cancer cells [19]. NEAT1 can function to be oncogenic by sponging the tumor-suppressive microRNAs. Study from Zhen et al., 2016 showed that NEAT1 promoted glioma pathogenesis by interacting with miR-449b-5p/c-Met axis [20]. NEAT1 was also found to promote laryngeal squamous cell cancer through regulating miR-107/CDK6 pathway [21]. In the breast cancer, NEAT1 is required for survival of breast cancer cells via targeting miR-548 [22]. In terms of chemo-resistance, NEAT1 was found to up-regulated EGCG-induced CTR1 to enhance cisplatin sensitivity in lung cancer cells [23]. These results may suggest that targeting NEAT1 may be beneficial for the treatment of human cancers. However, the role of NEAT1 in other non-studied types of cancer may be further investigated to confirm the role of NEAT1.

In the present study, the meta-analysis results implied that high NEAT1 expression was significantly associated with poor prognosis in patients with different types of cancers. The pooled HRs results showed that increased NEAT1 expression was positively associated with a shorter OS in patients with different types of cancers, which suggests the prognostic role of NEAT1 in predicting OS in cancer patients. Further subgroup analysis for OS showed that increased NEAT1 in cancer patients may be a reliable prognostic factor for hepatogastroenterol cancers. Apart from the included studies for solid tumors, NEAT1 was also found to have regulatory role in leukemia [24], however, the lack of relevant clinical data precluded it from meta-analysis in the present 
study. Apart from the function role NEAT1 in caner, NEAT1 also had other functional roles. For instances, NEAT1 was found to contribute to the pathogenesis of lupus [25]; altered expression of NEAT1 was also found in the Huntington's disease [26]; NEAT1 is required for mammary gland development and lactation [27], which suggests the diverse functional roles of NEAT1.

In the included studies from present study, only one study from Li et al., 2015 reported the association between increased NEAT1 expression and DFS in

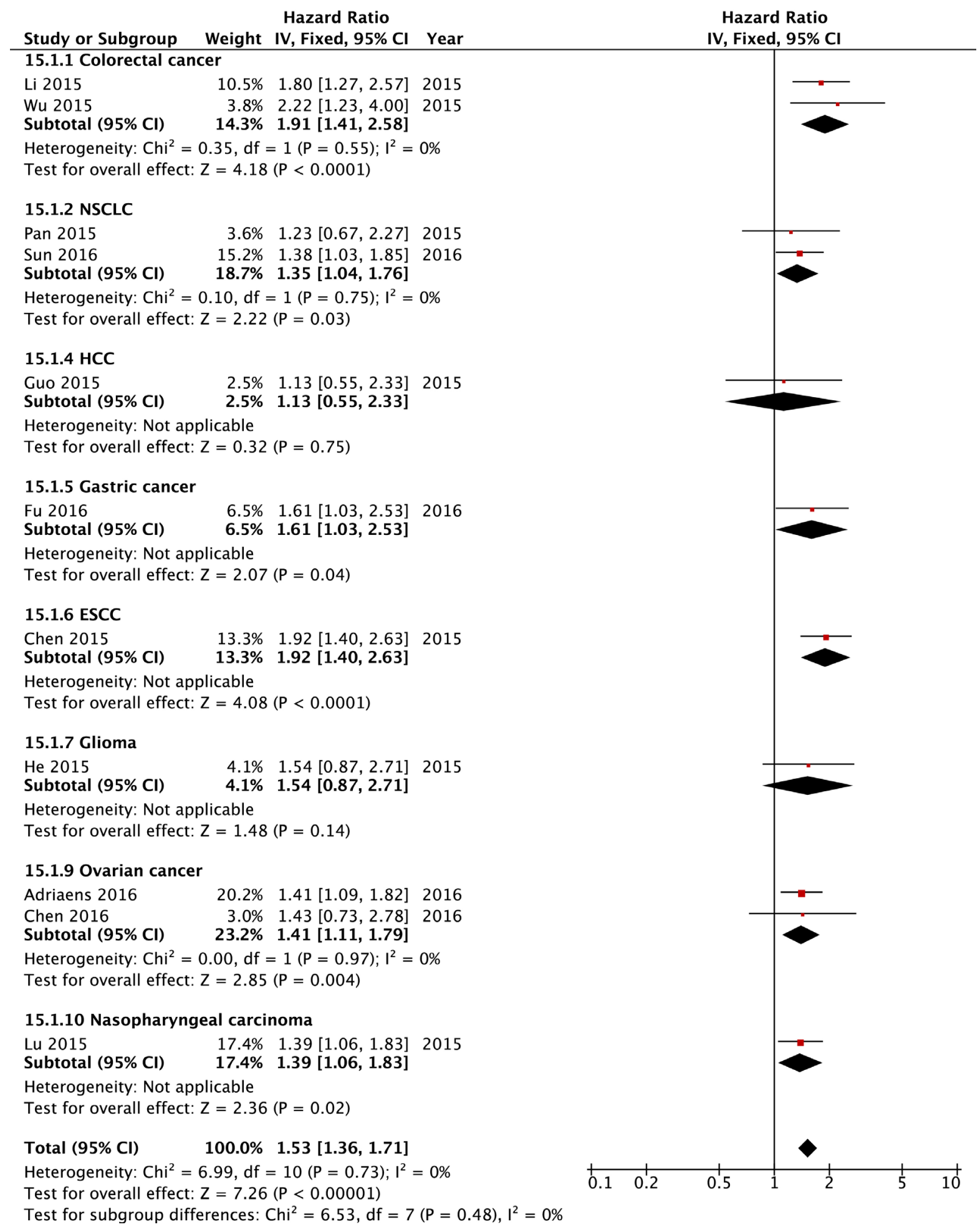

Figure 3: Forest plot of HRs for the association between high NEAT1 expression and OS in cancer patients stratified by different cancer types. 
colorectal cancer [11], and only one study from Adriaens et al., 2016 reported the association between increased NEAT1 expression and PFS in ovarian cancer [19], and thus meta-analysis was not performed in these two studies. However, these studies suggested the prognostic role of NEAT1 for DFS as well as PFS in these cancer patients. The meta-analysis for the association between increased NEAT1 expression and clinicopathological parameters was also analyzed in this study, and our results showed that increased NEAT1 expression was significantly associated with larger tumor size, lymph node metastasis, advanced TNM stage, poor tumor differentiation, distant metastasis, and higher invasion depth, which may suggest that increased NEAT1 may be associated with advanced features of cancer.

However, there are still some limitations in our meta-analysis from this study. For example, the total sample size was relatively small, and most of the patients included in the meta-analysis were from China. In addition, publication bias may exist, despite the fact that no significant publication bias was observed based on stable results revealed in sensitivity analysis as well as funnel plot analysis. Finally, the cut-off values definition for high NEAT1 expression was not consistent among the included. Therefore, larger-size, multi-center and higherquality studies with unified criteria for determining NEAT1 expression are necessary to solidify the results in this study.

In conclusion, the meta-analysis results suggest the prognostic role NEAT1 in prognosis in the patients with different types of cancer. However, due to several limitations of the included studies, larger-sample size, multi-center and higher-quality studies with consistent criteria for defining high NEAT1 expression level and low NEAT1 expression level may be required to further confirm the current findings in this study.

\section{MATERIALS AND METHODS}

\section{Literature search}

To retrieve potentially eligible studies, comprehensive literature search was performed in the following databases: PubMed, Web of Science, Embase, and CNKI, and the cut-off date was defined as September 30, 2016. The keywords for the search in these databases included: "nuclear enriched abundant transcript 1", "NEAT1", "long non-coding RNA NEAT1", "IncRNA NEAT1", "cancer", "tumor", "carcinoma", "neoplasm", and other eligible literatures were also manually evaluated from the references lists.

\section{Inclusion and exclusion criteria}

Inclusion criteria for the eligible studies included: (a) associations of NEAT1 expression levels with prognosis or clinicopathological features were described, (b) the role of NEAT1 in human cancer development was examined, (c) patients were categorized into two groups based on high and low expression levels of NEAT1, (d) the expression levels of NEAT1 in the cancer patients were determined by qRT-PCR. Exclusion criteria for the articles included: (a) studies without presenting data with relevant values, (b) duplicated publications, (c) letters, reviews, case reports and expert opinions.

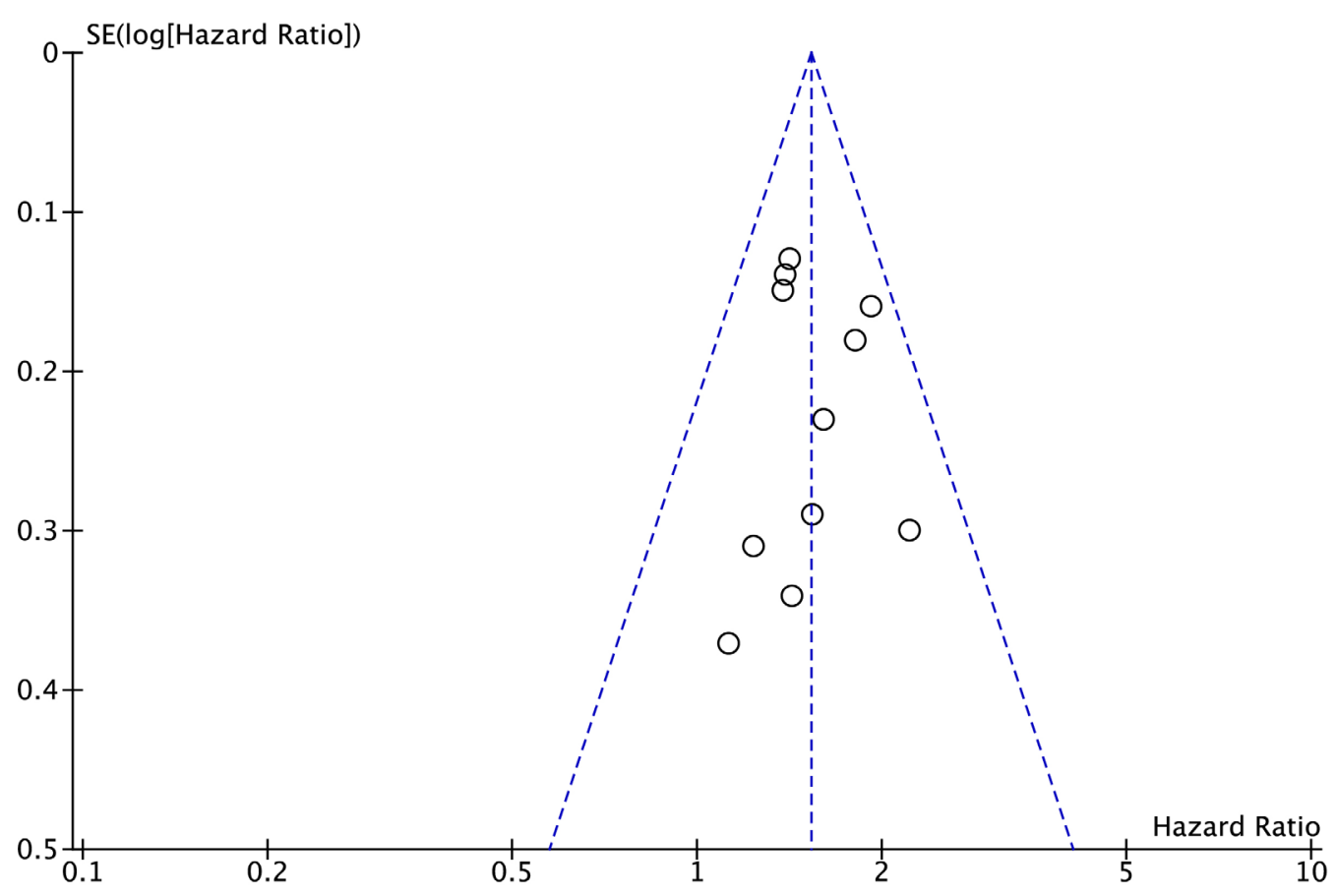

Figure 4: Funnel plot analysis for potential publication bias among included eligible studies. 


\section{Data extraction and quality assessment}

The data and information from all included eligible studies were independently evaluated by two investigators ( $\mathrm{ZL}$ and $\mathrm{YL}$ ). The following information were extracted from each eligible study: the name of first author, year of publication, cancer type, total number of patients from each eligible study, TNM stage, follow-up period, outcome measures, method for detecting NEAT1 expression, determination method, hazard ratio and its corresponding $95 \%$ confident interval, the clinicopathological parameters from each eligible study. For the eligible studies that provided both the univariate and multivariate analysis, the multivariate values were chosen as the multivariate values had higher precision on interpreting confounding factors. In the eligible studies only reporting Kaplan-Meier curves, the software, Enguage Digitizer (Version 4.1) was used to extract the survival data. In the situation of a disagreement, a consensus was reached by a third investigator (RX). The quality of all the included studies were assessed by The Newcastle-Ottawa Scale (NOS) method. The NOS scores ranged from 0 to 9 , and a study with an NOS score more than 6 was regarded as high quality.

\section{Statistical methods}

The meta-analysis was performed with RevMan 5.3 software and Stata SE12.0. The heterogeneity between studies was determined by the Chi square-based $Q$ test and $\mathrm{I}^{2}$ statistics. $P<0.05$ for the $Q$ test $\left(\mathrm{P}_{\mathrm{h}}\right)$ and $\mathrm{I}^{2}>50 \%$ were considered to be significantly heterogeneous. The fixed effects model was applied in the studies with no obvious heterogeneity $\left(\mathrm{P}_{\mathrm{h}}>0.05, \mathrm{I}^{2}<50 \%\right)$; the random effects model was applied in the studies with obvious heterogeneity $\left(\mathrm{P}_{\mathrm{h}} \leq 0.05, \mathrm{I}^{2} \geq 50 \%\right)$. The sensitivity analysis was also carried out to assess the stability of the results. A $P$ values less than 0.05 was considered to be statistically significant.

\section{ACKNOWLEDGMENTS AND FUNDING}

Thanks for the grant support from Special Research Fund from the Department of Education of Shaanxi Province (No.12JK07674).

\section{CONFLICTS OF INTEREST}

All the authors declared that there are no conflicts of interest.

\section{REFERENCES}

1. Wu S, Powers $\mathrm{S}, \mathrm{Zhu} \mathrm{W}$, Hannun YA. Substantial contribution of extrinsic risk factors to cancer development. Nature. 2016; 529:43-47.
2. Russnes HG, Lonning PE, Borresen-Dale AL, Lingjaerde OC. The multitude of molecular analyses in cancer: the opening of Pandora's box. Genome biology. 2014; $15: 447$.

3. Han Li C, Chen Y. Small and Long Non-Coding RNAs: Novel Targets in Perspective Cancer Therapy. Current genomics. 2015; 16:319-326.

4. Hombach S, Kretz M. Non-coding RNAs: Classification, Biology and Functioning. Advances in experimental medicine and biology. 2016; 937:3-17.

5. Jing W, Zhu M, Zhang XW, Pan ZY, Gao SS, Zhou H, Qiu SL, Liang CZ, Tu JC. The Significance of Long Noncoding RNA H19 in Predicting Progression and Metastasis of Cancers: A Meta-Analysis. BioMed research international. 2016; 2016:5902678.

6. Yan J, Zhou X, Dang Y, Yin C, Zhang G. Prognostic role of the long non-coding RNA metastasis-associated lung adenocarcinoma transcript 1 in various cancers: A metaanalysis. Molecular and clinical oncology. 2016; 4:100-106.

7. Serghiou S, Kyriakopoulou A, Ioannidis JP. Long noncoding RNAs as novel predictors of survival in human cancer: a systematic review and meta-analysis. Molecular cancer. 2016; 15:50.

8. Liu F, Zhu P, Luo H, Zhang Y, Qiu C. Prognostic value of long non-coding RNA UCA1 in human solid tumors. Oncotarget. 2016; 7:57991-58000. doi: 10.18632/oncotarget.11155.

9. Liu FT, Zhu PQ, Luo HL, Zhang Y, Hao TF, Xia GF, Zhu ZM, Qiu C. Long noncoding RNA ANRIL: a potential novel prognostic marker in cancer: a meta-analysis. Minerva medica. 2016; 107:77-83.

10. Liu FT, Xue QZ, Zhu ZM, Qiu C, Hao TF, Zhu PQ, Luo HL. Long noncoding RNA PVT1, a novel promising biomarker to predict lymph node metastasis and prognosis: a metaanalysis. Panminerva medica. 2016; 58:160-166.

11. Li Y, Li Y, Chen W, He F, Tan Z, Zheng J, Wang W, Zhao Q, Li J. NEAT expression is associated with tumor recurrence and unfavorable prognosis in colorectal cancer. Oncotarget. 2015; 6:27641-27650. doi: 10.18632/oncotarget.4737.

12. Chakravarty D, Sboner A, Nair SS, Giannopoulou E, Li R, Hennig S, Mosquera JM, Pauwels J, Park K, Kossai M, MacDonald TY, Fontugne J, Erho N, et al. The oestrogen receptor alpha-regulated lncRNA NEAT1 is a critical modulator of prostate cancer. Nature communications. 2014; 5:5383.

13. Sun C, Li S, Zhang F, Xi Y, Wang L, Bi Y, Li D. Long non-coding RNA NEAT1 promotes non-small cell lung cancer progression through regulation of miR-377-3p-E2F3 pathway. Oncotarget. 2016; 7:51784-51814. doi: 10.18632/ oncotarget.10108.

14. Fu JW, Kong Y, Sun X. Long noncoding RNA NEAT1 is an unfavorable prognostic factor and regulates migration and invasion in gastric cancer. Journal of cancer research and clinical oncology. 2016; 142:1571-1579.

15. Clemson CM, Hutchinson JN, Sara SA, Ensminger AW, Fox AH, Chess A, Lawrence JB. An architectural role for 
a nuclear noncoding RNA: NEAT1 RNA is essential for the structure of paraspeckles. Molecular cell. 2009; 33:717-726.

16. Bond CS, Fox AH. Paraspeckles: nuclear bodies built on long noncoding RNA. The Journal of cell biology. 2009; 186:637-644.

17. Souquere S, Beauclair G, Harper F, Fox A, Pierron G. Highly ordered spatial organization of the structural long noncoding NEAT1 RNAs within paraspeckle nuclear bodies. Molecular biology of the cell. 2010; 21:4020-4027.

18. Adriaens C, Marine JC. NEAT1-containing Paraspeckles: central hubs in stress response and tumor formation. Cell cycle (Georgetown, Tex). 2016:0.

19. Adriaens C, Standaert L, Barra J, Latil M, Verfaillie A, Kalev P, Boeckx B, Wijnhoven PW, Radaelli E, Vermi W, Leucci E, Lapouge G, Beck B, et al. p53 induces formation of NEAT1 lncRNA-containing paraspeckles that modulate replication stress response and chemosensitivity. Nature medicine. 2016; 22:861-868.

20. Zhen L, Yun-Hui L, Hong-Yu D, Jun M, Yi-Long Y. Long noncoding RNA NEAT1 promotes glioma pathogenesis by regulating miR-449b-5p/c-Met axis. Tumour biology : the journal of the International Society for Oncodevelopmental Biology and Medicine. 2016; 37:673-683.

21. Wang $\mathrm{P}, \mathrm{Wu} \mathrm{T}$, Zhou H, Jin Q, He G, Yu H, Xuan L, Wang X, Tian L, Sun Y, Liu M, Qu L. Long noncoding RNA NEAT1 promotes laryngeal squamous cell cancer through regulating miR-107/CDK6 pathway. Journal of experimental \& clinical cancer research. 2016; 35:22.

22. Ke H, Zhao L, Feng X, Xu H, Zou L, Yang Q, Su X, Peng L, Jiao B. NEAT1 is Required for Survival of Breast Cancer Cells Through FUS and miR-548. Gene regulation and systems biology. 2016; 10:11-17.

23. Jiang $P$, Wu $X$, Wang $X$, Huang W, Feng Q. NEAT1 upregulates EGCG-induced CTR1 to enhance cisplatin sensitivity in lung cancer cells. Oncotarget. 2016; 7:4333743351. doi: 10.18632/oncotarget.9712.

24. Blume CJ, Hotz-Wagenblatt A, Hullein J, Sellner L, Jethwa A, Stolz T, Slabicki M, Lee K, Sharathchandra A, Benner A, Dietrich S, Oakes CC, Dreger P, et al. p53dependent non-coding RNA networks in chronic lymphocytic leukemia. Leukemia. 2015; 29:2015-2023.

25. Zhang F, Wu L, Qian J, Qu B, Xia S, La T, Wu Y, Ma J, Zeng J, Guo Q, Cui Y, Yang W, Huang J, et al. Identification of the long noncoding RNA NEAT1 as a novel inflammatory regulator acting through MAPK pathway in human lupus. Journal of autoimmunity. 2016.
26. Sunwoo JS, Lee ST, Im W, Lee M, Byun JI, Jung KH, Park KI, Jung KY, Lee SK, Chu K, Kim M. Altered Expression of the Long Noncoding RNA NEAT1 in Huntington's Disease. Molecular neurobiology. 2016.

27. Standaert L, Adriaens C, Radaelli E, Van Keymeulen A, Blanpain C, Hirose T, Nakagawa S, Marine JC. The long noncoding RNA Neat1 is required for mammary gland development and lactation. RNA (New York, NY). 2014; 20:1844-1849.

28. Chen ZJ, Zhang Z, Xie BB, Zhang HY. Clinical significance of up-regulated lncRNA NEAT1 in prognosis of ovarian cancer. European review for medical and pharmacological sciences. 2016; 20:3373-3377.

29. Guo S, Chen W, Luo Y, Ren F, Zhong T, Rong M, Dang Y, Feng Z, Chen G. Clinical implication of long non-coding RNA NEAT1 expression in hepatocellular carcinoma patients. International journal of clinical and experimental pathology. 2015; 8:5395-5402.

30. He C, Jiang B, Ma J, Li Q. Aberrant NEAT1 expression is associated with clinical outcome in high grade glioma patients. APMIS : acta pathologica, microbiologica, et immunologica Scandinavica. 2016; 124:169-174.

31. Pan LJ, Zhong TF, Tang RX, Li P, Dang YW, Huang SN, Chen G. Upregulation and clinicopathological significance of long non-coding NEAT1 RNA in NSCLC tissues. Asian Pacific journal of cancer prevention : APJCP. 2015; 16:2851-2855.

32. Chen X, Kong J, Ma Z, Gao S, Feng X. Up regulation of the long non-coding RNA NEAT1 promotes esophageal squamous cell carcinoma cell progression and correlates with poor prognosis. American journal of cancer research. 2015; 5:2808-2815.

33. Wu Y, Yang L, Zhao J, Li C, Nie J, Liu F, Zhuo C, Zheng Y, Li B, Wang Z, Xu Y. Nuclear-enriched abundant transcript 1 as a diagnostic and prognostic biomarker in colorectal cancer. Molecular cancer. 2015; 14:191.

34. Lu Y, Li T, Wei G, Liu L, Chen Q, Xu L, Zhang K, Zeng D, Liao R. The long non-coding RNA NEAT1 regulates epithelial to mesenchymal transition and radioresistance in through miR-204/ZEB1 axis in nasopharyngeal carcinoma. Tumour biology : the journal of the International Society for Oncodevelopmental Biology and Medicine. 2016. 